Apidologie, 1987, 18 (2), 199-214

\title{
POLLENANALYSE, EIN HILFSMITTEL BEIM STUDIUM DES SAMMELVERHALTENS VON WILDBIENEN (HYMENOPTERA, APOIDEA)
}

\author{
Paul WESTRICH und Konrad SCHMIDT \\ Bezirkstelle für Naturschutz und Landschaftspflege, Nauklerstr. 58, D-7400 Tübingen \\ Zoologisches Institut der Universität, Kornblumenstr. 13, D-7500 Karlsruhe
}

\section{ZUSAMMENFASSUNG}

Mehr als 3300 Pollenladungen und Brutzelleninhalte von insgesamt 270 Bienenarten wurden lichtund zum Teil auch rasterelektronenmikroskopisch untersucht. Dadurch können Pollen-Spezialisten (oligolektische Arten) und -Generalisten (polylektische Arten) unterschieden werden, was anhand einiger Beispiele erläutert wird. Oligolektisch sind : Andrena agilissima und Osmia brevicornis an Brassicaceae, Andrena curvungula und Melitta haemorrhoidalis an Campanula (Campanulaceae), Andrena lathyri an Vicia und Lathyrus (Fabaceae), Andrena proxima an Apiaceae, Andrena vaga an Salix (Salicaceae), Chelostoma florisomne an Ranunculus (Ranunculaceae), Osmia cerinthidis an Cerinthe (Boraginaceae), Tetralonia alticincta an Asteraceae. Als polylektisch erwiesen sich: Andrena combinata, Anthophora bimaculata und Osmia cornuta. Sowohl bei oligolektischen wie bei polylektischen Arten zeigen die Analysen alle Übergänge zwischen hoher Blütenstetigkeit und der Nutzung mehrere Pollenquellen während eines Sammelfluges. Die Ergebnisse der Pollenanalysen werden mit den oft sehr diffusen Literaturangaben über «Futterpflanzen » verglichen, die Spezialisierungen (Oligolektie) oft nicht erkennen lassen. Zur zweifelsfreien Feststellung der Oligolektie ist die Pollenanalyse ein unverzichtbares Hilfsmittel.

\section{EINLEITUNG}

Pollen bildet den essentiellen Bestandteil der Larvennahrung von Wildbienen, der stets mehr oder weniger Nektar, in seltenen Fällen Öl, beigemischt ist. Schon seit langer Zeit ist bekannt, daß manche Bienenarten beim Pollensammeln die Blüten vergleichsweise weniger und immer wieder derselben Pflanzenarten besuchen, während ihr Blütenspektrum beim Nektarerwerb wesentlich weiter ist (vgl. BaKer und Hurd, 1968 ; Eickwort und Ginsberg, 1980 ; Linsley, 1958). Für dieses Pollensammeln zur Verproviantierung der Brutzellen werden seit RoBERTSON (1925) die Begriffe oligolektisch bzw. polylektisch verwendet. Als oligolektisch werden nach LinSLEY und Macswain 
(1957) die Arten bezeichnet, deren Weibchen im gesamten Verbreitungsgebiet auch beim Vorhandensein anderer Pollenquellen ausschließlich Pollen einer Pflanzenart oder nah verwandter Pflanzenarten sammeln und - wenn überhaupt - nur dann andere Pollenquellen nutzen, wenn die artspezifischen Pollenquellen ausfallen. Die Oligolektie ist im Normalfall auf Arten einer oder zweier Pflanzengattungen oder einer Pflanzenfamilie beschränkt. Oligolektische Bienenarten sind also Nahrungsspezialisten, vergleichbar mit den oligophagen Raupen vieler Schmetterlingsarten. Polylektische Arten verhalten sich gegenteilig, zeigen beim Pollensammeln also keine Bindung an bestimmte, nah verwandte Pflanzenarten.

Streng zu trennen von der vermutlich genetisch fixierten Oligolektie ist die sogenannte Blütenstetigkeit, die sich immer auf einzelne Individuen bezieht, während die Oligolektie ein Charakteristikum einer Bienenart ist. Da sich mit der Pollenanalyse nur das Pollensammeln erfassen läßt, verstehen wir unter der Blütenstetigkeit einer Wildbiene die «Treue»zu den Blüten einer einzigen Pflanzenart während eines Pollensammelfluges.

Während die «klassische» Blütenökologie im wesentlichen die Bedürfnisse der Pflanzen (Bestäubungsbiologie) untersucht, geht unser Ansatz in erster Linie von den Ansprüchen der Bienen aus. Die Bedürfnisse der beiden Organismengruppen müssen sich nicht decken, was schon DaRwIN (1876) feststellte. Bienen zeigen viel häufiger eine Spezialisierung auf bestimmte Blütenpflanzen als umgekehrt Pflanzen auf bestimmte Bestäuber (BAKER und HuRD, 1968). In zahlreichen Fällen liegen einseitige Bindungen (Bienen an Blüten) vor. Blütenbesuche sind daher nicht grundsätzlich Ausdruck einer Blüte-Bestäuber-Beziehung. So ist vermutlich außer Nektar- auch Pollendiebstahl weiter verbreitet als bisher bekannt ist.

\section{MATERIAL UND METHODE}

Zur Untersuchung kamen über 3300 Präparate von Pollenladungen und Brutzelleninhalten von 270 Bienenarten, wobei die Hummeln (Bombus) für die vorliegende Arbeit keine Berücksichtigung fanden. Der größte Teil (ca. $80 \%$ ) stammt von Wildbienen aus öfentlichen und privaten Sammlungen, dic zwischen 1846 und 1986 gefangen worden waren. Von 385 Exemplaren (ca. $11 \%$ ) wurde der gesammelte Pollen direkt im Gelände oder nach dem Abtöten im Labor entnommen. Außerdem wurde der Larvenproviant von 257 Brutzellen von 27 Arten (ca. $8 \%$ ) analysiert.

Dic Anfertigung der Pollenpräparate für die Licht- und Rasterclcktronenmikroskopie und die Untersuchungsmethoden sind an anderer Stelle ausführlich beschrieben (WESTRICH und SCHMIDT, 1986). Die Identifizierung der Pollen erfolgte mit Hilfe der Literatur (Moore und Webb, 1978; Punt, 1976; Punt und Clarke, 1980, 1981, 1984 ; SaWyer, 1981 ; Zander, 1935) und anhand von Vergleichspräparaten (Pollenherbar).

Der größtc Teil der Präparate wurde von Westrich im Rahmen des Forschungsprojektes «Wildbicnen in Baden-Württemberg " angefertigt, das vom Ministerium für Ernährung, Landwirtschaft, Umwelt und Forsten Baden-Württemberg aus Mitteln des Zweiten Untersuchungs- und Forschungsprogramms gefördert wird. 
ERGEBNISSE

Hier kann nur ein deiner Teil der Ergebnisse exemplarisch b shrieben werden. Eine ausführliche Darstellung wird an anderer Stelle erfolgen. Die Reihenfolge der Gattungen und Arten ist alphabetisch.

\section{Andrena agilissima (SCOPOLI) :}

15 Pollenladungen aus Deutschland, Italien und Spanien bestanden ausschließlich aus Pollen von Brassicaceae (Abb. 2d). Eine Pollenladung enthielt außerdem etwa zur Hälfte Pollen von Crataegus (Rosaceae). Im Feld haben wir die Art in verschiedenen Teilen Europas ausschließlich auf Brassicaceen pollensammelnd beobachtet (z.B. Sinapis arvensis, Brassica rapa, Barbarea vulgaris, Raphanus raphanistrum).

\section{Andrena combinata (CHRIST) :}

16 Pollenladungen aus Deutschland (BRD), Frankreich, Österreich und der Schweiz enthielten Pollen von 10 Pflanzenfamilien: Asteraceae, Apiaceae, Brassicaceae, Caryophyllaceae, Cistaceae, Crassulaceae, Fabaceae, Plantaginaceae, Ranunculaceae, Rosaceae. 4 Pollenladungen wiesen nur Pollen einer Pflanzenart auf, die übrigen setzten sich aus dem Pollen von 2-5 Pflanzenarten aus 2-4 Pflanzenfamilien zusammen.

\section{Andrena curvungula THOMSON :}

In 38 Pollenladungen aus Deutschland (BRD, DDR), Österreich, Polen, Jugoslawien, Belgien, Frankreich und Italien fand sich ausschließlich Pollen von Campanula (Campanulaceae). Davon bestanden 2 aus dem Pollen von 2 Campanula-Arten. Im Feld wurde die Art von uns ausschließlich an Campanula-Blüten pollensammelnd festgestellt (z.B. Campanula persicifolia, Campanula glomerata).

\section{Andrena lathyri AlfKen :}

46 Pollenladungen aus Deutschland (BRD, DDR), Österreich, Polen und Griechenland enthielten ausschließlich Pollen von Vicia und/oder Lathyrus (Fabaceae) (Abb. 2b). 3 Pollenladungen waren Mischladungen aus jeweils einer Art dieser beiden Gattungen. Die $q$ wurden von uns in verschiedenen Teilen Mitteleuropas stets an Vicia- oder Lathyrus-Arten beim Pollensammeln beobachtet (z.B. Vicia sepium, Vicia cracca, Lathyrus vernus, Lathyrus linifolius). 

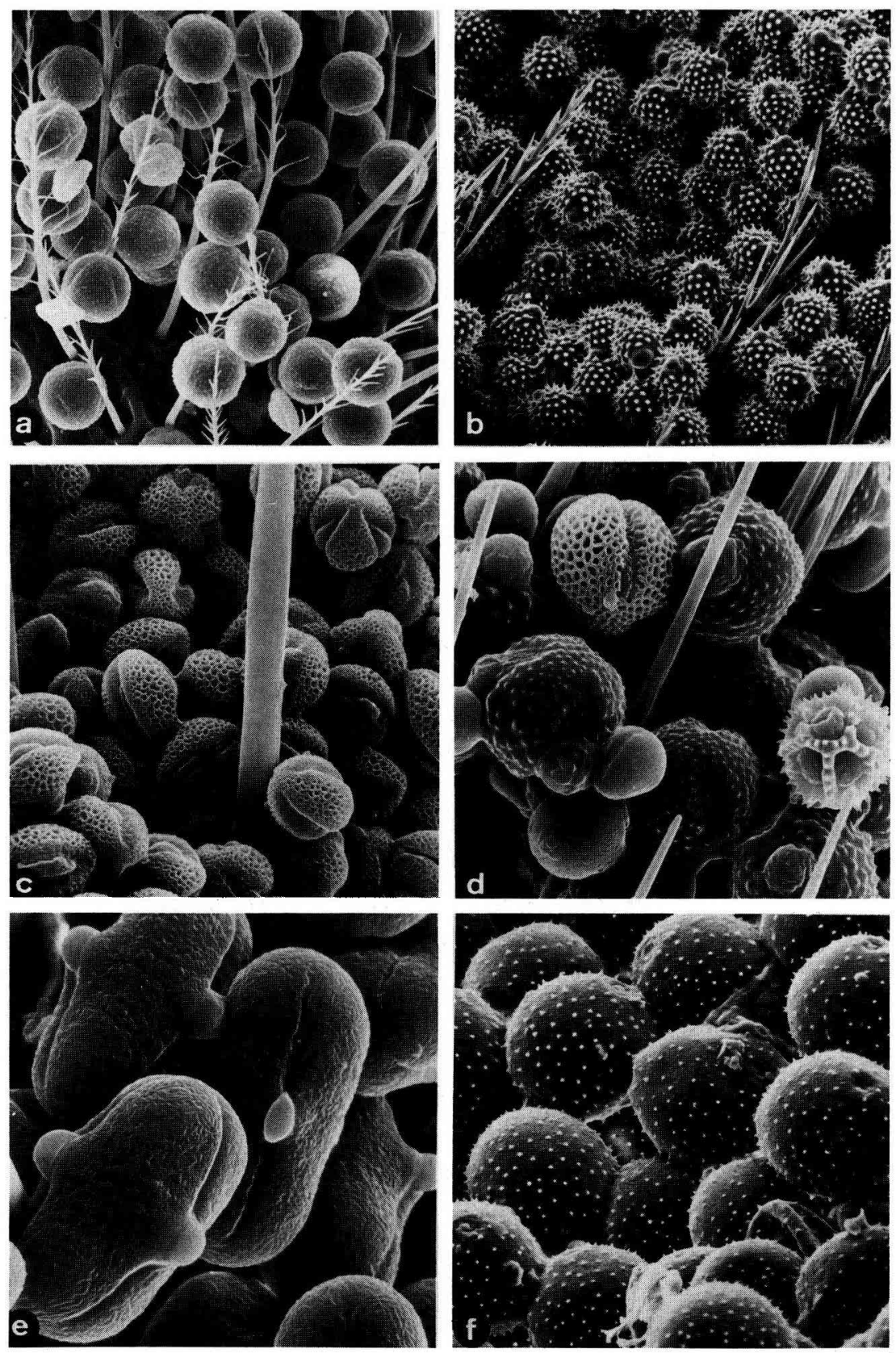
Andrena proxima (KIRBY) :

39 Pollenladungen aus Deutschland, England, Frankreich, Österreich, Ungarn und Italien bestanden ausschließlich aus Pollen von Apiaceae, überwiegend vom Anthriscus-Typ (Abb. 1e). 6 Pollenladungen enthielten Pollen von 2 Apiaceen-Arten. Die $q$ wurden von uns in verschiedenen Teilen Mitteleuropas nur an Apiaceen beim Pollensammeln beobachtet (z.B. Anthriscus sylvestris, Aegopodium podagraria, Heracleum sphondylium).

\section{Andrena vaga PANZER :}

16 Pollenladungen von verschiedenen Orten Süddeutschlands enthielten ausschließlich Pollen von Salix (Salicaceae) (Abb. 1c). Pollensammelnde $q$ wurden von uns nur an Salix-Arten beobachtet (z.B. Salix purpurea, Salix alba). Auch bei reichem Blütenangebot in unmittelbarer Nähe der Nester (Hochwasserdamm bei Karlsruhe) flogen pollensammelnde $q$ über Wald und Wasser mehr als $100 \mathrm{~m}$ zu den nächsten Salix-Beständen.

Авв. 1, a-f.

Fig. 1, a-f.

(a) Chelostoma florisomne (L.), Bauchbürste, Ranunculus-Typ, $500 x$

(a) Chelostoma florisomne (L.), Ventral scopa, Ranunculus type, 500x Funddaten (FD) : Karlsruhe, 13.6.1984, an Ranunculus Collection data (CD) : Karlsruhe, June 6, 1984, on Ranunculus

(b) Tetralonia alticincta (LEP.), Tibia, Helianthus-Typ, $500 x$

(b) Tetralonia alticincta (LEP.), Tibia, Helanthius type, $500 x$

FD : Umgebung Lurs/Provence, 1.-10.8.1983 an Pulicaria

CD : Near Lurs/Provence, Oct. 1-10, 1983 on Pulicaria

(c) Andrena vaga PZ., Tibia, mit Äther entfettet, Salix-Typ, $1040 x$

(c) Andrena vaga PZ., Tibia, fat removed with ether, Salix type, 1040x

FD : Karlsruhe, 5.5.1985, am Nest

CD : Karlsruhe, May 5, 1985, at the nest

(d) Anthophora bimaculata (PZ.), Tibia, $5 x$ Echium-Typ (kleinster Pollen),

$1 x$ Brassicaceae-Typ (Oberfläche genetzt), $1 x$ Taraxacum-Typ (durchbrochene Stachelkugel),

$11 x$ Centaurea jacea-Typ (warzig), 1x unbekannter Typ. Vergr. 1020x

(d) Anthophora bimaculata (PZ.), Tibia, 5x Echium type (smallest pollen), 1x Brassicaceae type (surface reticular), 1x Taraxacum type (perforated spine globe), 11x Centaurea jacea type (papillary), $1 x$ undefined type. Magnif. 1020x

FD : Mainz, 3.7.1966, an Echium vulgare

CD : Mainz, July 3, 1966, on Echium vulgare

(e) Andrena proxima (K.), Tibia, Apiaceae-Typ, $2280 x$

(e) Andrena proxima (K.), Tibia, Apiaceae type, 2280x

FD : Castelbuono, Sizilien, 19.5.1981

CD : Castelbuono, Sicily, May 19, 1981

(f) Melitta haemorrhoidalis (F.), Metatarsus, Campanula-Typ, $1000 x$

(f) Melitta haemorrhoidalis (F.), Metatarsus, Campanula type, 1000x

FD : Ruine Fleckenstein/Elsaß, 6.8.1984, an Campanula

CD : Ruines of Fleckenstein/Alsace, Aug. 6, 1984, on Campanula 
Anthophora bimaculata (PANZER) :

9 Pollenladungen aus Deutschland (BRD) enthielten Pollen von 9 Pflanzenfamilien : Asteraceae, Boraginaceae, Brassicaceae, Campanulaceae, Fabaceae, Hypericaceae, Lamiaceae, Lythraceae, Rosaceae (Abb. 1d). Alle waren Mischladungen aus dem Pollen von 2-5 Arten aus 2-4 Pflanzenfamilien. Auch die Feldbeobachtungen zeigten, daß sich die pollensammelnden $q$ wenig blütenstet verhalten. Bei heterogener Zusammensetzung der Vegetation in der Umgebung der Nester besuchten die $q$ auf ihren Sammelflügen die jeweils gerade blühenden Pflanzen in willkürlicher Reihenfolge.

\section{Chelostoma florisomne (LinNAEUs) (maxillosum L.) :}

Der Larvenproviant von 46 Brutzellen und 3 Pollenladungen, alle aus Süddeutschland, enthielten nur Pollen von Ranunculus (Ranunculaceae) (Abb. 1a). Der ausschließliche Ranunculus-Besuch pollensammelnder $q$ wird durch hunderte von eigenen Feldbeobachtungen in verschiedenen Teilen Süddeutschlands bestätigt.

\section{Melitta haemorrhoidalis (FABRICIUS) :}

27 Pollenladungen aus Deutschland (BRD, DDR), Österreich, Ungarn, Frankreich und England enthielten ausschließlich Pollen von Campanula (Campanulaceae) (Abb. 1f), davon bestanden 2 aus dem Pollen zweier Campanula-Arten. Bei hunderten von Feldbeobachtungen wurden pollensammelnde $\Varangle$ nur an Campanula-Arten festgestellt (z.B. Campanula rotundifolia, Campanula rapunculoides, Campanula trachelium).

\section{Osmia brevicornis (FABRICIUS) (atrocaerulea SchILlING) :}

Der Larvenproviant in 4 Nestern sowie 71 Pollenladungen aus Deutschland (BRD, DDR), Luxemburg, Polen, Schweiz, Österreich, Ungarn, Griechenland, Frankreich, Italien und Spanien enthielten ausschließlich Pollen von Brassicaceae. Davon bestanden 8 aus dem Pollen von 2 Brasssicaceen-Arten. 3 Pollenladungen aus Sipplingen (Bodensee) und Freiburg i.Br. enthielten außerdem bis zu 1/3 Pollen von Ranunculus.

\section{Osmia cerinthidis MorawITz :}

20 Pollenladungen aus Deutschland (BRD), Österreich, Ungarn, Tschechoslowakei und Türkei enthielten ausschließlich Pollen von Cerinthe (Boraginaceae) (Abb. 2c). Vermutlich wird der Cerinthe-Pollen vom $q$ zunächst mit der korbförmigen Clypealarea, die durch zwei seitliche hornartige Fortsätze und einen Haarkranz umgrenzt ist, gesammelt und anschließend in die Bauch- 
bürste (Transporteinrichtung) gebracht. Bei mehreren der untersuchten $q$ war die Clypealarea mit Cerinthe-Pollen gefüllt. Eigene Feldbeobachtungen liegen nicht vor.

Osmia cornuta (LATREILLE) :

Der Larvenproviant aus 40 Brutzellen sowie 6 Pollenladungen enthielt Pollen von 6 Pflanzenfamilien: Aceraceae, Asteraceae, Liliaceae, Papaveraceae, Rosaceae und Salicaceae. Der Larvenproviant in einer Brutzelle bestand jeweils entweder aus dem Pollen einer Pflanzenart oder von 2-3 Pflanzenarten aus 2-3 Pflanzenfamilien. 3 Pollenladungen wiesen nur Pollen einer einzigen Pflanzenart auf (Abb. 2a). Feldbeobachtungen in Süddeutschland bestätigen das weite Spektrum der Pollenquellen dieser Art. Die $q$ wurden von uns an Vertretern von 11 verschiedenen Pflanzenfamilien pollensammelnd festgestellt.

Tetralonia alticincta (LEPELETIER) :

4 Pollenladungen aus Deutschland, Frankreich und Spanien enthielten ausschließlich Pollen von Pulicaria (Asteraceae) (Abb. 1b). Im Feld (Südfrankreich, Nordspanien) haben wir die $q$ in verschiedenen Jahren stets nur an Pulicaria dysenterica pollensammelnd beobachtet.

\section{DISKUSSION}

Die Analyse der Pollenladungen und Brutzelleninhalte ermöglicht eine kritische Bewertung der Blütenbesuchsangaben, die bisher in faunistischen, blütenökologischen und ethologischen Arbeiten von den verschiedensten Autoren gemacht wurden. Pollenquellen können von (gelegentlich zusätzlich besuchten) austauschbaren Nektarquellen unterschieden werden. Einige Beispiele mögen dies näher erläutern.

Andrena agilissima besucht nach Pitrioni und Schmidt (1943) in Niederösterreich besonders Reseda lutea (Resedaceae) und Raphanus (Brassicaceae). KNUTH (1898-1905) zählt auf : Cheiranthus cheiri, Brassica rapa (Brassicaceae), Reseda lutea (Resedaceae), Melilotus altissima (Fabaceae), Fragaria vesca (Rosaceae), Crepis biennis (Asteraceae), Thymus serpyllum (Lamiaceae), Salix cinerea, Salix caprea, Salix aurita (Salicaceae). Kocourex (1966:85) nennt ohne Erläuterung außer einigen Brassicaceae noch Reseda lutea (Resedaceae), Ribes (Grossulariaceae), Helianthemum (Cistaceae), Syringia vulgaris (Oleaceae), Taraxacum officinale (Asteraceae) und Myosotis (Boraginaceae). Die Pollenanalyse beweist aber, daß Andrena agilissima eine oligolektische, auf Brassicaceae spezialisierte Art ist. 

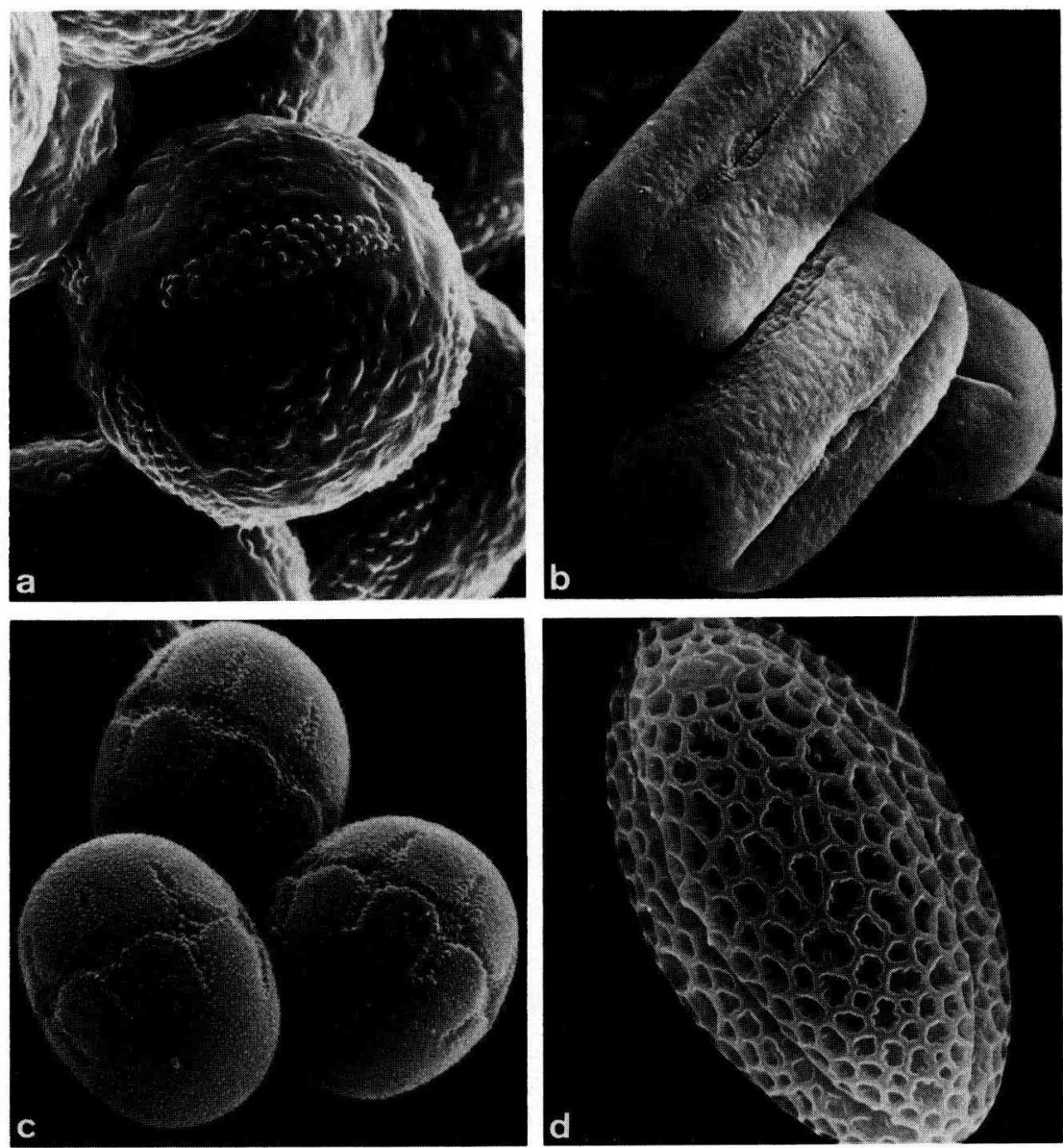

AвB. 2, a-d.

FIG. 2, a-d.

(a) Osmia cornuta (LATR.), Bauchbürste, Corydalis solida-Typ, 2300x

(b) Osmia cornuta (LATR.), Ventral scopa, Corydalis solida type, 2300x

FD : Tübingen, 10.4.1979, an Corydalis cava CD : Tübingen, April 10, 1979, on Corydalis cava

(b) Andrena lathyri ALFK., Tibia, Vicia-Typ, 2100x

(b) Andrena lathyri ALFK., Tibia, Vicia type, 2100x

FD : Umgebung Wels, Österreich, 16.5.1983

CD : Near Wels, Austria, May 16, 1983

(c) Osmia cerinthidis MOR., Clypealarea, Cerinthe-Typ, 3900x

(c) Osmia cerinthidis MOR., Clypealarea, Cerinthe type, $3900 x$

FD : Umgebung Linz, Österreich, 11.5.1968

CD : Near Linz, Austria, May 11, 1968

(d) Andrena agilissima (SCOP.), Metatarsus, Brassicaceae-Typ, 3000x

(d) Andrena agilissima (SCOP.), Metatarsus, Brassicaceae type, 3000x

FD : Mallorca, Spanien, 4.1963

CD : Mallorca, Spain, April 1963 
Für Andrena curvungula nennt KocoureK (1966: 87) folgende Blütenbesuche : Campanula (Campanulaceae), Lavatera (Malvaceae), Geranium (Geraniaceae), Veronica chamaedrys (Scrophulariaceae), Laserpitium (Apiaceae) und Aquilegia vulgaris (Ranunculaceae). Nach KRATоchwil (1984:600) besucht diese Art " ausschließlich Campanula- und Geranium-Arten ». Die Spezialisierung dieser oligolektischen Art auf Campanula-Arten als Pollenquellen wird aber durch die Pollenanalysen eindeutig bewiesen. Geranium-Arten werden von $\sigma^{\prime}$ und $q$ als (austauschbare) Nektarquellen genutzt, von den $q$ jedoch nie als Pollenquellen. Überhaupt haben Geranium-Arten für die Populationsentwicklung mitteleuropäischer Wildbienen keinerlei Bedeutung. Der leicht kenntliche Geranium-Pollen wurde nur in insgesamt 8 Pollenladungen ausgesprochen polylektischer Arten gefunden, stets mit nur geringem prozentualem Anteil. Von Geranium sanguineum, dem Kratochwil (1984:639) eine hohe Bedeutung für Bienen beimißt, konnten wir nur einzelne Pollenkörner bei einem zum Nest heimkehrenden Weibchen von Halictus quadricinctus feststellen, obwohl in der Umgebung des Nistplatzes (Vogtsburg im Kaiserstuhl) reichlich Geranium sanguineum blühte. Oligolektisch und auf Campanula spezialisiert ist Melitta haermorrhoidalis.

Andrena lathyri besucht nach StoeckнerT (1933:150) in Franken fast ausschließlich Lathyrus linifolius (Fabaceae), nach Kocourek (1966:52) in der Tschechoslowakei Lathyrus vernus, Lathyrus linifolius, Vicia sepium, Trifolium repens (Fabaceae), Ajuga reptans, Thymus serpyllum (Lamiaceae), Symphytum officinale (Boraginaceae) und Salix (Salicaceae). Die zu enge Einschätzung durch StовскнеRT beruht vermutlich auf Beobachtungen in einem zu eng begrenzten Gebiet, KocoureK reiht undifferenziert beobachtete Blütenbesuche aneinander. Andrena lathyri ist aber aufgrund der Pollenanalyse charakterisiert als eine oligolektische, auf Vicia und Lathyrus spezialisierte Art. KratochwIL (1984 : 600) schreibt : « Tritt fast ausschließlich an rot-violetten Lathyrus-Arten auf, sie meidet aber z.B. den gelbblühenden Lathyrus pratensis ». BrauN (1985) fing aber an Lathyrus pratensis ein $q$, dessen einheitliche Pollenladung er Lathyrus pratensis zuschreibt.

Andrena proxima besucht nach StOeckHERT (1933:145) «mit Vorliebe Umbelliferen (= Apiaceae), sowie Euphorbia cyparissias, Taraxacum officinale, Ranunculus bulbosus und Bellis perennis». Von Kratochwil (1984:604) wurde die Art «zwar nicht an Umbelliferen, jedoch ausschließlich an Euphorbia cyparissias gefunden $»$. Durch die Pollenanalysen ist Andrena proxima aber zweifelsfrei als eine oligolektische, auf Apiaceae spezialisierte Art gekennzeichnet.

Ebenfalls oligolektisch sind Andrena vaga, die auf Salix und Osmia cerinthidis, die auf Cerinthe spezialisiert ist. Die Oligolektie des auf Ranunculus (Ranunculaceae) spezialisierten Chelostoma florisomne wird durch unsere und 
durch die von KÄPYLÄ (1978) in Finnland gemachten Analysen belegt. Tetralonia alticincta dürfte ebenso eine oligolektische, auf Asteraceae spezialisierte Art sein, auch wenn von ihr bisher nur wenige Pollenladungen mangels Material zur Untersuchung kommen konnten. Von nächstverwandten Vertretern der Artengruppe von Tetralonia ruficornis (FABRICIUS) werden als besuchte Blüten nur Asteraceae (Inula-Arten) genannt (TKalcô, 1979).

Für Osmia brevicornis gibt Westrich $(1983: 25)$ als Blütenbesuch an : «Picris, Crepis». Zahlreiche andere Autoren nennen ebenfalls u.a. Asteraceae. Von Radchenko (1978) untersuchte Nester waren « meist mit Pollen von Erysimum canescens (Brassicaceae) gefüllt». Pesenko (1971) beobachtete die Art vorwiegend auf Brassicaceae und führt sie in einer Liste von Nahrungsbeziehungen (Pesenko, 1975) unter dieser Pflanzenfamilie an. Unsere Pollenanalysen bestätigen die Beobachtungen der russischen Autoren und beweisen, daß Osmia brevicornis eine oligolektische, auf Brassicaceae spezialisierte Art ist.

Die Polylektie von Osmia cornuta wird nicht nur durch unsere, sondern auch durch die von TASEI (1973) in Frankreich gemachten Pollenanalysen bewiesen, so daß bei dieser Art die vielfältigen Blütenbesuchsangaben der Autoren nicht überraschen. Auch für Anthophora bimaculata werden in der Literatur Vertreter mehrerer Planzenfamilien aufgezählt. Die Pollenanalyse belegt die ausgesprochene Polylektie dieser Art.

In der Literatur sind unter den Begriffen «Blütenbesuch », « Futterpflanzen » oder "Anflugpflanzen" in der Regel alle möglichen Beobachtungen ohne nähere Differenzierung zusammengefaßt. Darauf weist bereits KäPYLä (1978) hin. So wird meist nicht zwischen $\sigma^{\prime}$ und $q$ und bei den $q$ nicht zwischen Nektarbesuchen zur Eigenversorgung und Pollensammeln für die Brut unterschieden. Dies hat bis in die jüngste Zeit zu Fehlinterpretationen blütenökologischer Beziehungen geführt (z.B bei Kratochwil, 1983, 1984). Aus demselben Grund sind aus der Literatur Spezialisierungen (Oligolektie) oft nicht zu erkennen, obwohl die spezifischen Pollenquellen in der Liste der besuchten Pflanzenarten meist mit aufgeführt sind. Die verschiedenen Zwecke der Blütenbesuche, die wir selbst früher nicht ausreichend unterschieden haben, wurden von uns an anderer Stelle dargestellt (Westrich und Schmidt, 1986). Wenn bei oligolektischen Arten außer den spezifischen Pollenquellen von den Autoren weitere Pflanzenarten genannt werden, so dürften wohl meist Blütenbesuche zur Eigenversorgung mit Nektar beobachtet worden sein. Blütenbesuche von $\sigma^{\prime \prime}$ sind zur Ermittlung von Spezialisierungen (Oligolektie) untauglich, da die $\sigma^{\prime \prime}$ oligolektischer Arten mehr oder weniger oft auch an solchen Blüten Nektar saugen, die von den $q$ nie zum Pollensammeln aufgesucht werden.

Bei $\nmid$ sind gravierende Fehleinschätzungen vor allem dann möglich, wenn sie pollenbeladen an Blüten angetroffen werden. Eine exakte Beobachtung des 
Blütenbesuchsverhaltens läßt aber in den meisten Fällen eine Unterscheidung zwischen Pollensammeln und reinem Nektarerwerb zu. Denn auch oligolektische $q$ können auf ihrem Pollensammelflug untypische Nektarquellen (z.B. auf dem Heimweg) aufsuchen. So fand Braun (1985) bei einem $q$ von Andrena proxima, das von ihm auf Crepis capillaris (Asteraceae) gefangen wurde, nur Pollen von Aegopodium podagraria und Heracleum sphondylium, zwei Vertretern der Pflanzenfamilie Apiaceae, auf die diese Bienenart spezialisiert ist. Ein $\Varangle$ von Andrena lathyri, von BRAUN auf Rhinanthus minor (Scrophulariaceae) gefangen, trug nur Pollen von Vicia sepium, also eines Vertreters einer der beiden Fabaceen-Gattungen, auf die diese Bienenart spezialisiert ist. Zahlreiche weitere Beispiele ähnlicher Art ließen sich hier noch anfügen.

Einzelne Pollenladungen oligolektischer Bienenarten enthalten neben « typischem » Pollen zusätzlich noch Pollen von Pflanzenarten, die von der Art normalerweise nicht zum Pollensammeln besucht werden. Einzelne $q$ weichen gelegentlich, aber nicht immer, auf andere Pollenquellen aus, wenn die artspezifischen Pollenquellen durch natürliche oder menschliche Einwirkungen ausfallen. In allen Fällen kehren die Bienen sofort zu ihren normalen Pollenquellen zurück, sobald diese wieder verfügbar sind (TноrP, 1969 ; Westrich, unpubl.). Die Bindung an bestimmte Pollenquellen kann aber auch so stark sein, daß die Verproviantierung der Brutzellen hinausgezögert oder sogar ganz eingestellt wird, wenn die Pollenquellen noch nicht blühen oder (im Experiment) entfernt werden (Bohart und Youssef, 1977 ; EICKwort, 1973 ; STrickler, 1979).

Teilpopulationen oder einzelne $q$ sonst polylektischer Arten können lokal und/oder zeitweise eine hohe Blütenstetigkeit zeigen und dann dem Beobachter Oligolektie vortäuschen. Dies kann vor allem dann auftreten, wenn nur eine oder wenige Pflanzenarten blühen und daher das Angebot an Pollenquellen eingeschränkt ist, oder wenn einzelne Pflanzenarten besonders ergiebige Pollenquellen darstellen. KRatochwil (1984: 604) schreibt Andrena combinata eine Präferenz für Apiaceen zu, vermutlich aufgrund einer lokalen Beobachtung und unkritischer Übernahme von Literaturangaben. Wie die Analysen der Pollenladungen von $q$ verschiedener Fundorte jedoch zeigen, ist keine Bindung an Apiaceen nachweisbar, da es sich bei dieser Art um eine ausgesprochen polylektische handelt.

Die Pollenanalyse ermöglicht auch die Kontrolle der Blütenstetigkeit von \& während eines Pollensammelfluges bzw. während der gesamten Verproviantierung einer Brutzelle. Die untersuchten Pollenladungen zeigen alle Übergänge zwischen hoher Blütenstetigkeit und der Nutzung mehrerer Pollenquellen während eines Sammelfluges und dies bei oligolektischen wie bei polylektischen Arten. Eine wichtige Voraussetzung der Blütenstetigkeit dürfte die Blütendichte einer Pollenquelle sein. Oligolektische Bienen unterscheiden bei ihren Sammelflügen vielfach nicht zwischen gleichzeitig blühenden, nahver- 
wandten Arten derselben Pflanzengattung bzw. Pflanzenfamilie, müssen sich aber zwangsläufig hoch blütenstet verhalten, wenn nur eine spezifische Pollenquelle blüht. Daher beruht der früher verwendete Begriff « monolektisch » auf einem Artefakt und ist zu verwerfen. Auf der anderen Seite zeigen auch die $q$ ausgesprochen polylektischer Arten zeitweise eine hohe Blütenstetigkeit, sogar während der gesamten Verproviantierung einer Brutzelle (z.B. Osmia cornuta, Osmia rufa).

Manchmal wird versucht, bestimmte Beziehungen zwischen Bienengattungen oder gar -familien und Blütentypen bzw. Pflanzengesellschaften verallgemeinernd zu beschreiben. So ist nach Paulus (1978) Bauchsammeln von Pollen " zweifellos in direkter Anpassung an sternotribe Antherenlage entstanden und umgekehrt». Entsprechend sollen Megachile-Arten bevorzugt an Fabaceen-Blüten Pollen sammeln. Innerhalb der einzelnen Megachile-Arten finden sich tatsächlich aber ganz unterschiedliche Bevorzugungen, wie über 200 untersuchte Pollenladungen zeigen. Das Spektrum reicht von oligolektischen bis hin zu ausgesprochen polylektischen Arten. Eine solche Beurteilung auf Gattungsebene ist also nicht möglich. KRATOCHwIL (1983) behandelt das Blütenbesuchsverhalten ausgesprochen artenreicher und biologisch sehr vielfältiger Bienengattungen wie Andrena und Osmia ebenfalls auf Gattungsebene und versucht Bevorzugungen dieser Gattungen für bestimmte Pflanzengesellschaften (Rasen, Säume) aufzuzeigen. Seine Aussagen basieren außerdem oft auf wenigen Einzelbeobachtungen stets an einer einzigen Lokalität. Zwar wurden Literaturangaben verarbeitet, daß diese aber nicht unkritisch übernommen werden dürfen, wurde bereits weiter oben erläutert. KRATOCHWIL unterscheidet außerdem nicht zwischen Kuckucksbienen und nestbauenden Arten, nicht zwischen $\sigma^{\prime}$ und $q$ und bei den $q$ nestbauender Arten wiederum nicht zwischen Blütenbesuchen zur Eigenversorgung und Pollensammelbesuchen. Nach allen bisherigen Erkenntnissen ist aber für die Populationsentwicklung einer Bienenart neben einem artspezifischen Nistplatz und - oft austauschbaren - Nektarquellen von ausschlaggebender Bedeutung, ob das Angebot an spezifischen Pollenquellen gering oder reichlich ist. Bevor also bei der Diskussion von Landschaftspflegemaßnahmen Forderungen zur Erhaltung bestimmter Sukzessionsstadien, z.B. versaumter Halbtrockenrasen (KRATOCHWIL, 1983) erhoben werden oder theoretische Überlegungen zur «Co-Evolution» bestimmter Pflanzengesellschaften und Bienen angestellt werden (КTRATOCHwIL, 1984), müssen zuerst die Spezialisierungen (Oligolektie) auf Artebene einwandfrei geklärt und berücksichtigt werden. Hierfür ist die Pollenanalyse ein unverzichtbares Hilfsmittel. 


\section{DANK}

Für die großzügige Erlaubnis, von Sammlungstieren Pollenladungen abzunehmen und für vielfältige Unterstützung danken wir den Herren : J. Braun (Klingenmünster), Dr. F. Brechtel (Rülzheim), Dr. E. Diller (Zoologische Staatssammlung, München), D. Doczkal (Malsch), A.W. Ebmer (Linz, Österreich), G.R. Else (British Museum (Natural History), London), Dr. W. Grünwaldt (München), Mag. F. Gusenlettner (Oberösterreichisches Landesmuseum, Linz), Dr. H. Hohmann (Überseemuseum Bremen), Dr. F. Косн (Zoologisches Museum Berlin, DDR), Dr. J.P. KopelKe (Forschungsinstitut Senckenberg, Frankfurt a.M.), Br. V. Lefeber (Maastricht, Holland), Dr. P. Lögler (Naturkundemuseum, Freiburg i.Br.), Dr. T. Osren (Staatliches Museum für Naturkunde, Stuttgart), Dr. D.S. Peters (Forschungsinstitut Senckenberg, Frankfurt a.M.), H. Riemann (Bremen), Prof. Dr. U. RoesLer (Landessammlungen für Naturkunde, Karlsruhè), M. Schwarz (Ansfelden, Österreich), Dr. E. SreinmanN (Chur, Schweiz).

Für methodische und Literaturhinweise, die Hilfe bei der Untersuchung schwieriger Präparate und die Möglichkeit das Pollenherbar zu benutzen, haben wir Dr. G. VoRwoHL (Landesanstalt für Bienenkunde, Stuttgart-Hohenheim) zu danken. Herr Prof. Dr. F. Habermalz (Institut f. Wissenschaftl. Mikroskopie der Univ. Tübingen) hat liebenswürdigerweise ein Mikroskop zur Verfügung gestellt. Den Herren Dipl. Phys. P. Pfundstein und W. Send (Physik. Institut d. Uni. Karlsruhe) danken wir für die Anfertigung der REM- Aufnahmen.

Frau L. Hofmann (Tübingen) danken wir für ihre Mithilfe bei der Herstellung von Pollenpräparaten, den Herren Prof. G.C. Eickwort (Ithaca, USA) und Dipl. Biol. Ch. Westerkamp (Mainz) für Literaturhinweise und kritische Diskussionen.

\section{RÉSUMÉ}

\section{L'ANALYSE POLLINIQUE, OUTIL POUR ÉTUDIER LE COMPORTEMENT DE BUTINAGE DES ABEILLES SAUVAGES (HYMENOPTERA, APOIDEA)}

On a étudié au microscope optique - et en partie au microscope électronique à balayage - plus de 3300 pelotes de pollen et contenus de cellules de couvain provenant de 270 espèces d'abeilles. On a pu ainsi différencier les spécialistes (espèces oligolectiques) des généralistes (espèces polylectiques) et donner quelques exemples à titre d'illustration. Les abeilles oligolectiques sont : Andrena agilissima et Osmia brevicornis sur les Brassicacées, Andrena curvungula et Melitta haemorrhoidalis sur Campanula (Campanulacées), Andrena lathyri sur Vicia et Lathyrus (Fabacées), Andrena proxima sur les Apiacées, Andrena vaga sur Salix (Salicacées), Chelostoma florisomne sur Ranunculus (Ranunculacées), Osmia cerinthidis sur Cerinthe (Boraginacées), Tetralonia alticincta sur les Asteracées. Andrena combinata, Anthophora bimaculata et Osmia cornuta sont polylectiques. Chez les oligolectiques comme chez les polylectiques, les analyses montrent toutes les transitions au cours d'un même vol entre la constance florale stricte et l'utilisation de plusieurs sources de pollen. Les résultats des analyses polliniques sont comparées avec les données de la littérature, souvent très diffuses, concernant les plantes alimentaires, où l'on ne peut souvent pas reconnaître les spécialisations (oligolectie). L'analyse pollinique est un moyen fort utile pour déterminer sans ambiguité l'oligolectie.

\section{SUMMARY}

\section{POLLEN ANALYSIS, AN AUXILIARY TOOL TO STUDY THE COLLECTING BEHAVIOR OF WILD BEES (HYMENOPTERA, APOIDEA)}

More than 3300 pollen loads and larval provisions of 270 bee species were analyzed using light and scanning electron microscopy. Pollen specialists (oligolectic species) and generalists (polylectic species) could thus be distinguished. Examples are given for both types of specialization. Oligoleges are : Andrena 
agilissima and Osmia brevicornis on Brassicaceae, Andrena curvungula and Melitta haemorrhoidalis on Campanula (Campanulaceae). Andrena lathyri on Vicia and Lathyrus (Fabaceae), Andrena proxima on Apiaceae, Andrena vaga on Salix (Salicaceac), Chelostoma florisomne on Ranunculus (Ranunculaceae), Osmia cerinthidis on Cerinthe (Boraginaccac), Tetralonia alticincta on Asteraceac. Polyleges are: Andrena combinata, Anthophora bimaculata and Osmia cornuta. The pollen loads of oligolectic and of polylectic species showed all degrees from high flower constancy to the use of several pollen sources during one collecting trip. The results of the pollen analyses were compared with flower visitation records in the literature, where specializations often are not to be recognized. Pollen analysis provides a very important means to discover host specifity in pollen collecting behavior.

\section{LITERATUR}

Baker H.G., Hurd P.D., 1968. - Intrafloral ecology. Annu. Rev. Entomol, 13, 385-414.

Bohart G.E., Youssef N.N., 1977. - The biology and behavior of Evylaeus galpinsiae. Wasmann J. Biol., 34 (1976), 185-234.

BrauN J., 1985. - Blütenökologische Untersuchungen an einem aufgelassenen Weinberg und einer Fettwiese bei Klingenmünster/Pfalz. Zulassungsarbeit Wiss. Prüfung Lehramt Gymn., Zool. Institut Univ, Karlsruhe.

Darwin C., 1876. - The Effects of Cross and Self-Fertilization in the Vegetable Kingdom. Murray, London.

Eickwort, G.C., 1973. - Biology of the European mason bee, Hoplitis anthocopoides (Hymenoptera : Megachilidae), in New York State. Search Agric (Cornell Univ. Agr. Exp. Sta.), 3 (2), 31 p.

Eickwort G.C., Ginsberg H.S., 1980. - Foraging and mating behavior in Apoidea. Annu. Rev. Entomol., 25, 421-446.

KÄPYLÄ M., 1978. - Bionomics of five wood-nesting solitary species of bees (Hym., Megachilidae), with emphasis on flower relationships. Biol. Research Reports Univ. Jyväskylä, 5, 3-89.

KNUTH P., 1898-1905. - Handbuch der Blütenbiologie. W. Engelmann. Leipzig, 3 Bde.

Kocourex M., 1966. - Prodromus der Hymenopteren der Tschechoslowakei, Pars 9 : Apoidea, 1. Acta faun. ent. Mus. nat. Pragae, 12, 1-122.

Kratochwil A., 1983. - Zur Phänologie von Pflanzen und blütenbesuchenden Insekten (Hymenoptera, Lepidoptera, Diptera, Coleoptera) eines versaumten Halbtrockenrasens im Kaiserstuhl - ein Beitrag zur Erhaltung brachliegender Wiesen als Lizenz-Biotope gefährdeter Tierarten. Beih. Veröff. Naturschutz Landschaftspflege Bad.-Württ., 34, 57-108.

Kratochwil A., 1984. - Pflanzengesellschaften und Blütenbesucher-Gemeinschaften : biozönologische Untersuchungen in einem nicht mehr bewirtschafteten Halbtrockenrasen (Mesobrometum) im Kaiserstuhl (Südwestdeutschland). Phytocoenologia, 11 (4), 455-669.

Linsley E.G., 1958. - The ecology of solitary bees. Hilgardia, 27, 543-599.

Linsley E.G., Macswain J.W., 1957. - The nesting habits, flower relationships, and parasites of some North American species of Diadasia. Wasmann J. Biol., 15, 199-235.

Moore P.D., WebB J.A., 1978. - An Illustrated Guide to Pollen Analysis. Hodder and Stoughton, London.

Paulus H.F., 1978. - Co-Evolution zwischen Blüten und ihren tierischen Bestäubern. Sonderbd. naturwiss. Ver. Hamburg, 2, 51-81.

Pesenko YU.A., 1971. - On the fauna and ecology of Apoidea (Hymenoptera) of the steppes of the Lower Don. 1. The Family Megachilidae. Entomol. Rev., 50, 38-47.

Pesenko YU.A., 1975. - On the fauna and ecology of Apoidea (Hymenoptera) of the Lower Don. 6. A review of the trophic links. Entomol. Rev., 54, 53-59. 
Pittion B., Schmidt R., 1943, - Die Bienen des südöstlichen Niederdonau. II. Andrenidae und isoliert stehende Gattungen. Niederdonau/Natur $u$. Kultur, 24, 1-83.

Punt W., (Hrsg.), 1976. - The Northwest European Pollen Flora. I. Elsevier, Amsterdam, Oxford, New York.

Punt W., Clarke G.C.S. (Hrsg.), 1980. - The Northwest European Pollen Flora. II. Elsevier, Amsterdam, Oxford, New York.

Punt W., Clarke G.C.S. (Hrsg.), 1981. - The Northwest European Pollen Flora. III. Elsevier, Amsterdam, Oxford, New York.

Radchenko V.G., 1978. - A new type of nest without cells in Metallinella atrocoerulea (Hymenoptera, Megachilidae). Entomol. Rev., 57, 353-355.

Robertson C., 1925. - Heterotropic bees. Ecology, 6, 412-436.

SAWYer R.W., 1981. - Pollen Identification for Beekeepers. Univ. College Cardiff Press, Cardiff.

Stoeckhert F.K., 1933. - Dic Bienen Frankens (Hym. Apid.). Eine ökologisch-tiergeographische Untersuchung. Beih. Dtsch. entomol. Z., 1932, 1-294.

Strickler K., 1979. - Specialization and foraging efficiency of solitary bees. Ecology, 60, 998-1009.

TASEI J.N., 1973. - Le comportement de nidification chez Osmia (Osmia) cornuta LATR. et Osmia (Osmia) rufa L. (Hymenoptera, Megachilidac). Apidologie, 4, 195-225.

ThовP R.W., 1969. - Systematics and ecology of bees of the subgenus Diandrena (Hymenoptera, Andrenidae). Univ. California Publ. Entomol., 52, 1-146.

TKalce B., 1979. - Revision der curopäischen Vertreter der Artengruppe von Tetralonia ruficornis (Fabricius) (Hymenoptera, Apoidea). Acta Mus. Moraviae, 64, 127-152.

Westrich P., 1983, - Dic Bicnen Baden-Württembergs I. Megachilidac (Hymenoptera, Apoidea). Suttgarter Beitr. Naturkunde, Ser. A, Nr. 363, 1-50.

Westrich P., Schmidt K., 1986. - Methoden und Anwendungsgebicte der Pollenanalyse bei Wildbienen (Hymenoptera, Apoidea). Linzer biol. Beitr., 18, 341-360.

Zander E., 1935. - Beiträge zur Herkunftsbestimmung bei Honig. I. Pollengestaltung und Herkunftsbestimmung bei Blütenhonig mit besonderer Berücksichtigung des deutschen Trachtgebietes. Reichsfachgruppe Imker, Berlin. 